\title{
Comparing Three Interaction Methods for Manipulating Thin Deformable Virtual Objects
}

\author{
Johannes Hummel, Robin Wolff; Andreas Gerndt ${ }^{\ddagger}$ \\ Software for Space Systems and Interactive Visualization \\ German Aerospace Agency (DLR), Germany
}

\author{
Torsten Kuhlen $\$$ \\ Virtual Reality Group \\ RWTH Aachen University, Germany
}

\begin{abstract}
We present results of a user study in which we compared three interaction methods for manipulating deformable objects in immersive virtual environments. The task was to control a virtual robot hand removing a thin foil cover from a satellite in an on-orbit servicing training simulator. The lack of haptic feedback placed a high challenge on the user when trying to apply the right force for grasping the foil without losing grip or damaging it. We compared the intuitiveness and effectiveness of using a tracked joystick, finger distance measurement, and a novel prototype enabling direct force input through pinching.
\end{abstract}

Index Terms: B.4.2 [Input/Output Devices]: Channels and controllers; H.3.4 [Systems and Software]: Performance evaluation H.5.2 [User Interfaces]: Interaction styles I.3.7 [Three-Dimensional Graphics and Realism]: Virtual Reality

\section{INTRODUCTION}

Simulating the realistic grasping of objects in immersive virtual environments is a great challenge. Although sophisticated motion tracking enables natural interaction with objects, a lack of haptic feedback makes it hard to judge whether an object is actually touched on the surface or penetrated. Physics simulation can be used to support grasping tasks by detecting collisions between a virtual hand and the grasped object. The computed resulting forces can then be used to move the objects accordingly. This, however, provides visual feedback only. Several interaction devices have been developed that provide haptic feedback. Most of them, however, have been designed for a desktop environment and offer only limited interaction workspace.

We compared three simple approaches for supporting the grasping of deformable objects using two commonly used standard input devices: a Flystick2 and a finger tracking device, as well as a novel prototype of a finger tracking device extended with pinch-sensitive analog input. The prototype was used in a related user study that compared finger-based direct interaction to controller-based ray interaction for grasping virtual objects [1]. Our focus was on providing a method for intuitively and precisely controlling an analog input value in immersive object manipulation tasks.

\section{USeR STUdy}

Our user study is based on an ongoing project that aims to develop a multi-modal virtual environment used for the analysis, training and programming of on-orbit servicing tasks, as well as to help to develop and examine new designs of serviceable satellites and servicing robots. It will be used to train astronauts in manned servicing

\footnotetext{
*e-mail: Johannes.Hummel@dlr.de

†e-mail: Robin.Wolff@dlr.de

†e-mail: Andreas.Gerndt@dlr.de

§e-mail: kuhlen@vr.rwth-aachen.de
}

IEEE Virtual Reality 2012

4-8 March, Orange County, CA, USA

978-1-4673-1246-2/12/\$31.00 @2012 IEEE missions, as well as to program and remotely operate service robots in space within unmanned missions.

We used the scenario of removing the protective multi-layer insulation (MLI) foil of satellites as a benchmark, in which the subjects had to control a virtual robot hand to carefully grasp and move the MLI without damaging it. A requirement was to avoid folds by slightly stretching it while moving it from one side to the other. The subjects had to apply a force, strong enough to pull the MLI away without letting it glide through the robot's fingers, but also not to grasp too hard destroying it. Thus, the challenge was to apply the appropriate force and holding it while moving the arm in a wide arc of approximately $1 \mathrm{~m}$ from right to left. The test scenario was implemented using a software framework [3] that provided the physics simulation of the robot hand and MLI, as well as supported our immersive display system (see Figure 1).

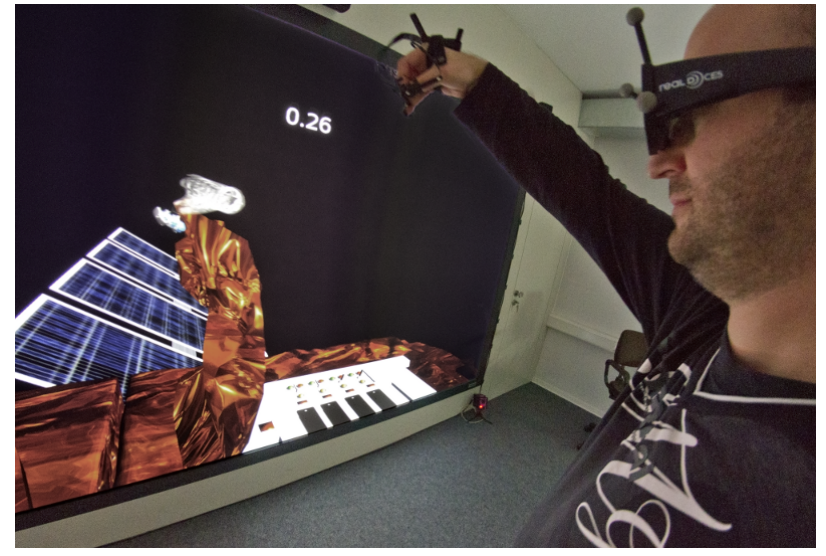

Figure 1: A user trying to remove the MLI with the pinch-sensitive finger tracking device.

\subsection{Interaction Devices and Interaction Methods}

The study was conducted in front of a $1.9 \times 3.5 \mathrm{~m}$ rear-projection system with active stereo visualization. Head-tracking was provided by a four-camera optical tracking system by A.R.T.. Its standard interaction device is the Flystick2. We linearly mapped the analog value of the joystick's y-axis to opening and closing of the virtual robot hand. Pushing the joystick up would open the hand, and pulling it down would close the hand. In idle position, the fingers were just before closed, so that the user had to pull down the joystick to apply the desired force.

In addition to the Flystick2, we used an off-the-shelf fingertracking device that tracked the position and orientation of the back of the hand and all five finger-tips. Force control was mapped linearly to the distance between the fingers and the thumb. When the user's hand was still open for about $1 \mathrm{~cm}$, the virtual one was already closed with the fingers and thumb touching each other. Moving the fingers closer than $1 \mathrm{~cm}$ would apply a force to the virtual hand.

Finally, we used a prototype based on the finger-tracking device, additionally equipped with electrodes measuring the skin impedance between fingers and thumb. This was used to detect 
not only the contact, but also the intensity of the contact. The intensity was used to linearly control the force applied to the fingers of the virtual robot hand. In order to support the subjects finding the appropriate force to be applied to the finger-tips of the virtual hand, a normalized value (between 0 and 1 ) was displayed in an overlay text on the screen, which turned green when the force was within the allowed range, red when it was above, and white otherwise.

\subsection{Subjects and Procedure}

Twelve people participated in our study. Eight of them had no to little experience interacting with VR devices. This group consisted mostly of engineers and satellite experts, resembling the end users of our training simulator. The remaining four people had medium to high VR experience. Each subject completed the task using the three interaction devices: Flystik2 (FS), finger-tracking with distance measurement (FT) and direct force input (FT-F). Each task was repeated three times. In order to counteract learning effects, we changed the order of devices between participants [2]. However, both finger tracking devices were always used in a row to avoid repeated calibration.

After a short introduction, the subjects were given a few minutes time to familiarize themselves with the system and the task before we started the measurements. During the trial, we measured the resulting force applied to the virtual hand, as well as the occurrences of applying the desired force, too little (MLI slipped out of the hand) or too much force (MLI got pinched or torn apart). After the trial, the subjects completed a questionnaire on intuitiveness, ease of use and preference of interaction method.

\section{Results and Discussion}

Figure 2 shows the number of successful and failed attempts of removing the MLI from the virtual satellite. On average, each subject solved two out of three trials successfully. No significant difference could be found between the conditions regarding the success rate. Looking closer at the causes for failed attempts reveals that using finger-tracking with force input (FT-F) the subjects often applied too much force, while they applied too little force when using the normal finger-tracking (FT) and Flystick2 (FS). On average, one to two attempts were necessary to properly grasp the MLI, while slightly more grasping attempts were observed in the FT-F condition.

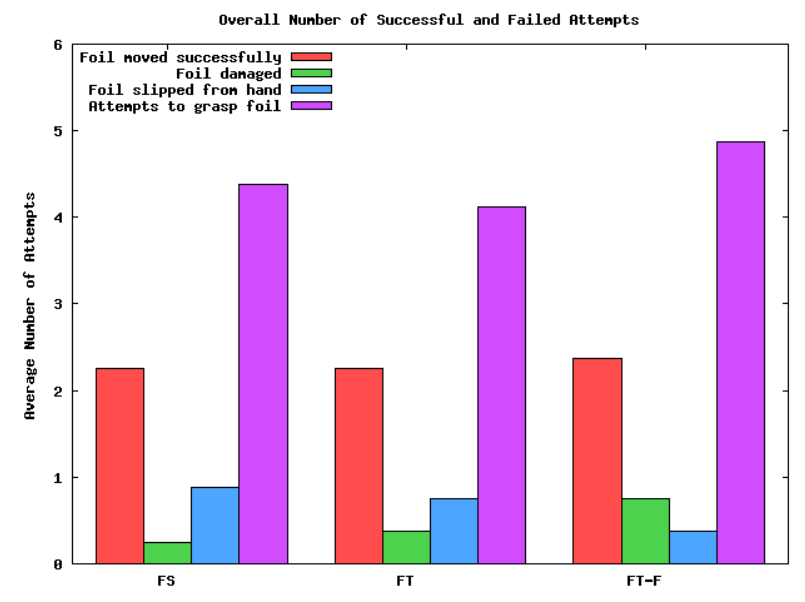

Figure 2: Average number of successful and failed attempts to move the foil, as well as number of attempts to grasp the foil.

Analyzing the recordings of applied forces (not shown in this paper), it turned out that using the Flystick 2 produced the most stable force values over the duration of moving the MLI. Continuously holding down the joystick with the thump while moving the arm appeared to be easy for most subjects. Presumably, this was supported by the damping effect of the joystick design. Using the normal finger-tracking device and controlling the applied forces via the distance between fingers and thumb showed the least deviation in all measurements. However, the distance between the fingers continuously increased while moving the arm in an wide arc, resulting in a slightly increasing force applied to the virtual hand. Most subjects appeared to have difficulties applying a constant force with the FT-F method while moving the grasped MLI. Our measurements showed a high deviation of force values in this condition. A reason for this might be the relatively small input range and high sensitivity of this device. Already a slight pinch was converted to a force and applied to the virtual hand, making it hard finding the desired pressure between the finger-tips. This, however, varied across the subjects due to different skin types and conductance.

Evaluating answers to the questionnaire, we found that, on one hand, the subjects considered the FT-F method as most intuitive to carefully grasp and move the MLI. It was followed by the FT method and finally FS (see Figure 3). On the other hand, the subjects stated that with the FT method it was less difficult to find and hold the desired force for solving the task than in the FS and the FT-F conditions. When asked which interaction method the subjects would choose if they had to solve the task again, 67\% answered they would choose FT-F, 25\% the FT method, while $8 \%$ would choose FS. This correlates with our results on intuitiveness, but slightly contradicts the overall subjects' opinion about the ease of using the interaction devices.
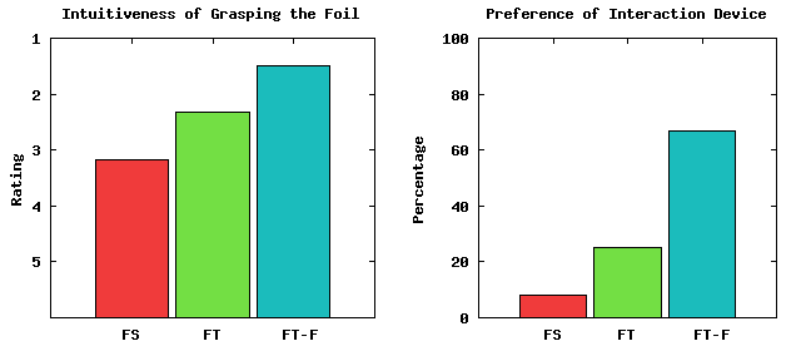

Figure 3: Left: Intuitiveness of using the interaction device for grasping and moving the foil. (Rating was from 1 =fully agree to 5 =fully disagree) Right: User preference of interaction device in percent.

\section{Conclusion ANd Future Work}

We compared three distinct interaction methods for user input when carefully manipulating thin, deformable objects in immersive virtual environments. Results showed that, although using a joystick and the finger-distance based method allowed more precise input, users preferred the direct pinch-sensitive input method. In our future work, we will try to improve the stability of the direct force input and will investigate further interaction methods performing similar tasks within two handed object manipulation tasks.

\section{ACKNOWLEDGEMENTS}

We wish to thank Advanced Realtime Tracking $\mathrm{GmbH}$ for providing us with their prototype and additional equipment for the study.

\section{REFERENCES}

[1] M. Moehring and B. Froehlich. Effective manipulation of virtual objects within arm's reach. In IEEE Virtual Reality, pages $131-138$, 2011.

[2] J. E. Swan. Experimental design and analysis for human-subject visualization experiments. IEEE Visualization Tutorial, 2008.

[3] R. Wolff, C. Preusche, and A. Gerndt. A modular architecture for an interactive real-time simulation and training environment for satellite on-orbit servicing. In IEEE International Symposium on Distributed Simulation and Real Time Applications, pages 72-80, 2011. 